\title{
Proposta de uma Forma Reduzida do WISC-IV para Avaliação Intelectual de Surdos ${ }^{1}$
}

\author{
Tharso de Souza Meyer², Vera Lúcia Marques de Figueiredo \\ Universidade Católica de Pelotas, Pelotas-RS, Brasil
}

RESUMO

Todo instrumento de avaliação deve ser revisado e adaptado quando utilizado em uma população diferente daquela para a qual foi padronizado. Este artigo apresenta os procedimentos teóricos do processo de adaptação de uma Forma Reduzida do teste WISC-IV para surdos. Objetivando uma avaliação mais rápida, porém representativa do modelo quadrifatorial do teste, foram selecionados oito subtestes: Vocabulário, Semelhanças, Cubos, Raciocínio Matricial, Código, Procurar Símbolos, Dígitos e Sequência de Números e Letras. As instruções e os itens foram traduzidos para Libras e a versão inicial foi apresentada à peritos na cultura surda e testada em oito alunos surdos. A forma experimental resultou em inclusão e exclusão de itens, inserção de ilustrações e itens de exemplos e, ainda, algumas modificações no processo de aplicação dos subtestes, atendendo às especificidades da cultura surda. Após os ajustes necessários, a versão experimental deve ser testada em uma amostra mais representativa para a realização das análises psicométricas.

Palavras-chave: WISC-IV, surdos, inteligência, adaptação transcultural, forma reduzida.

\section{ABSTRACT - WISC-IV Reduced Form Proposal for Intellectual Assessment of the Deaf}

Each assessment instrument should be reviewed and adapted when used with a population other than the one for which it was standardized. This article presents the theoretical procedures of the adaptation process for a Reduced Form of the WISC-IV test for deaf people. Aiming for a faster, but representative, evaluation of the quadripartite model of the test, eight subtests were selected: Vocabulary, Similarities, Cubes, Matrix Reasoning, Coding, Symbol Search, Digit Span, and Letter-Number Sequencing. Instructions and items were translated into sign language and the initial version was presented to experts in deaf culture and tested on eight deaf students. The experimental form resulted in inclusion and exclusion of items, insertion of illustrations and sample items, as well as some modifications in the process of applying the subtests, taking into account the specificities of the deaf culture. After the necessary adjustments, the experimental version should be tested in a more representative sample for performing the psychometric analysis. Keywords: WISC-IV; Deaf; Intelligence; Transcultural adaptation; Reduced form.

\section{RESUMEN - Propuesta de una Forma Reducida del WISC-IV para Evaluación Intelectual de Sordos}

Cada instrumento de evaluación debe ser revisado y adaptado cuando es utilizado en una población diferente de aquella para el cual fue creado. En este artículo se presentan los procedimientos teóricos del proceso de adaptación de una forma reducida del test WISC-IV para sordos. Fueron seleccionados ocho sub-tests con el objetivo de una evaluación más rápida pero también representativa del modelo cuatriparal del mismo: Vocabulario, Semejanzas, Diseño con Cubos, Raciocinio Matricial, Códigos, Búsqueda de Símbolos, Dígitos y Secuencia de Números y Letras. Las instrucciones y los ítems fueron traducidos para Libras y la versión inicial se presentó a peritos en cultura de sordos y fue experimentada en ocho estudiantes también sordos. La forma experimental del test resultó en inclusión y exclusión de ítems, inserción de ilustraciones e ítems de ejemplos y también algunas modificaciones en el proceso de aplicación de los sub-tests, de acuerdo a las características específicas de la cultura de sordos. Después de los ajustes necesarios, la versión experimental se debe probar en una muestra más representativa para la realización de los análisis psicométricos.

Palabras clave: WISC-IV; Sordos; Inteligencia; Adaptación Transcultural; Forma reducida.

\section{Introdução}

Os surdos apresentam uma cultura própria, uma vez que a forma de apreender o mundo é peculiar devido à sua singularidade. Como método de comunicação, esses indivíduos utilizam a Língua de Sinais
(LS), sendo, assim, considerada a língua materna dessa comunidade. Sua modalidade de articulação é visuoespacial, ao contrário das línguas faladas que são oral-auditivas. São utilizados recursos com as mãos, movimentos corporais e expressões faciais, sendo que as variações na gesticulação transmitem informações sobre

Agradecimentos: À Editora Casa do Psicólogo, pelo apoio e material fornecido, aos Tradutores e Intérpretes de Libras (TILS) Ana Marci Ferreira, Larissa Ramos, Joseane Vianna, Nádia Gonçalves, Daniel Silveira e Crisiane Soares e, também, ao Fabiano Rosa, pela valiosa colaboração na etapa de Análise de Juízes. À Escola Especial Professor Alfredo Dub (Pelotas-RS), em particular à diretora Marli Shulz, à coordenadora pedagógica Janeth Pierobom e à professora Ana Marci Ferreira, por terem aceitado participar do estudo, demonstrando interesse no desenvolvimento do conhecimento acerca da surdez.

Endereço para correspondência: Rua XV de Novembro, 457, apto 704, 96015-000, Pelotas-RS. Tel.: (53) 3225-0273. Cel.: (53) 99146-9739.

E-mail: tharso.psico@gmail.com 
as qualidades dos objetos ou detalhes dos movimentos (Lubbadeh, 2006).

Autores acreditam que a língua de sinais, além de favorecer os processos cognitivos responsáveis pela formação de conceitos, fornece todo o aparato linguístico necessário para a estruturação do pensamento e a aquisição de outras línguas, garantindo, assim, o pleno desenvolvimento do surdo (Goldfeld, 1997; Quadros, 1997). No Brasil, os surdos utilizam a Língua Brasileira de Sinais - Libras, oficializada em abril de 2002 (Lei 10.346).

Em nível internacional, as Escalas Wechsler de Inteligência são as mais utilizadas para a avaliação da inteligência. A vantagem de utilizar tais escalas em relação a outros testes consiste em poder identificar a inteligência cristalizada e fluida, além de avaliar habilidades cognitivas específicas por meio dos subtestes. Entretanto, os estudos da cognição dos surdos foram embasados, na sua maioria, na utilização da Escala de Execução (Anastasi \& Urbina, 2000; Braden, 1992; Braden \& Hannah, 1998; Maller, 1994; Marchesi, 2004).

Encontram-se pesquisas com a população de surdos envolvendo: as diferentes edições das Escalas Wechsler; os tipos de comunicação utilizada na administração do teste (linguagem oral, linguagem sinalizada e Língua de Sinais); as diferentes variáveis que podem influenciar no desenvolvimento cognitivo (pais surdos, tipo de escola, etc.) e as características psicométricas do teste (validade, fidedignidade e estrutura fatorial), além da criação de normas específicas para esse grupo (Braden, 1992; Maller, 1994; Maller, 1997). Apesar da maioria dos estudos priorizarem a aplicação do Conjunto de Execução, encontram-se também, alguns estudos com os subtestes verbais. Informações sobre o desempenho cognitivo de surdos foram incluídas no manual do WISC-III (Wechsler, 1991).

Krouse (2008) analisou as características psicométricas do teste WISC-IV utilizando dados de arquivo de crianças surdas avaliadas por psicólogos de nove Estados Americanos. Segundo a autora, os resultados sugerem que os Índices de Compreensão Verbal e Organização Perceptual do WISC-IV avaliaram os surdos com maior precisão do que as crianças da amostra de padronização ouvintes (Krouse, 2008).

A quarta edição da Escala Infantil - WISC-IV trouxe grandes mudanças em relação aos seus predecessores, sobretudo a ruptura da tradição hierárquica dos instrumentos de Wechsler na representação dos QIs Verbal e Execução, assimilados nos Índices de Compreensão Verbal e Organização Perceptual, respectivamente. Portanto, houve uma simplificação na Estrutura Fatorial, reformulação dos subtestes, redefinição dos construtos subjacentes e atualização do material quanto à portabilidade e apresentação visual (Vidal, Figueiredo \& Nascimento, 2011; Wechsler, 2013).

Historicamente, formas curtas da Escala de Inteligência Wechsler são utilizadas para atender à necessidade de um instrumento de avaliação rápida (Dumont \& Faro, 1993; Wagner Pawlowski, Yates, Camey, \& Trentini, 2010). Principalmente em função do grande tempo envolvido na aplicação, correção e na interpretação dessas Escalas, a necessidade de economia de tempo tem sido a maior justificativa para o desenvolvimento de formas reduzidas (Coutinho \& Nascimento, 2010). Essas formas vêm sendo tradicionalmente utilizadas em situações, como pesquisas de larga escala, em ocasiões de rastreio, situações de triagem, em reavaliações, em estudos com grupos clínicos ou em uma situação em que a investigação de um perfil cognitivo mais amplo não se faz necessário.

Na literatura estrangeira, já se encontram estudos sobre formas reduzidas do WISC-IV. Entre eles, o de Ryan, Glass e Brown (2007), que analisaram diferentes formas (com dois, três, quatro e seis subtestes) para a população ouvinte. Uma forma de sete subtestes foi desenvolvida por Crawford, Anderson, Rankin e McDonald (2010). Com base na amostra de padronização americana (ouvintes), os autores analisaram as características psicométricas e estimaram os QIs utilizando os subtestes: Cubos, Vocabulário, Raciocínio Matricial, Semelhanças, Dígitos, Procurar Símbolos e Código. Estes foram selecionados para serem representantes dos quatro Índices do WISCIV, sendo dois de cada fator, com exceção do Índice de Memória Operacional, com apenas um subteste. O subteste Sequência de Letras e Números não foi selecionado por mostrar maior dificuldade entre as crianças. Os autores concluíram que essa forma reduzida apresentou boas características psicométricas, tanto de validade como fidedignidade, e oferece os mesmos métodos de análise da versão completa (Crawford et al., 2010). Uma forma similar à de Crawford foi utilizada no presente estudo.

Ao propor uma forma reduzida do WISC-IV para surdos, deve-se levar em conta a necessidade de adaptar transculturalmente o instrumento, tendo em vista a ausência de ferramentas específicas para a avaliação intelectual dessa população. Conforme dito anteriormente, os surdos constituem uma cultura própria, que vai além da Língua de Sinais. Assim, diversos autores (Jorge, 1998; Pasquali et al., 2010; Borsa, Damásio \& Bandeira, 2012; Goresnstein, Wang, \& Hungerbühler, 2016) apontam a relevância da equivalência cultural, semântica, linguística e contextual.

O objetivo do presente artigo é apresentar a etapa dos procedimentos teóricos da adaptação de uma versão reduzida do WISC-IV para surdos. Dessa forma, os profissionais que trabalham com a comunidade surda, poderão dispor de uma ferramenta a ser testada para posterior uso no Brasil.

\section{Método}

Pasquali refere que o trabalho para elaborar ou validar um instrumento psicológico deve abranger três momentos: procedimentos teóricos, empíricos e analíticos (Pasquali et al. 2010; Goresnstein, Wang, \& 
Hungerbühler, 2016). O artigo refere-se ao primeiro procedimento, sendo realizado aqui em quatro etapas, descritas a seguir.

\section{Etapa de Seleção dos Subtestes para Compor a Forma Reduzida}

Conforme Vechia (2011), não há um modelo único de FR mais adequado. Na literatura existem pesquisas com diferentes arranjos, oscilando entre dois e oito subtestes. Todos os estudos enfatizam que, quanto maior o número de provas empregadas, menor o erro padrão de medida e maiores os coeficientes de validade e fidedignidade.

A seleção dos subtestes que compuseram a forma reduzida deste estudo baseou-se em três considerações buscadas na literatura: estudos em que foram avaliados sujeitos surdos com as diversas edições do WISC, estudos que verificaram a eficácia de FRs (abreviadas ou curtas) com as diversas edições do WISC e estudos que analisaram as características psicométricas dos subtestes nas amostras de padronização brasileiras (do WISC-III e WISC-IV). Ainda, foi considerado na escolha dos subtestes, o critério de terem pouco conteúdo verbal para evitar as dificuldades de adaptação para a Língua de Sinais.

\section{Etapa de Tradução e Adaptação para Libras}

Conforme Braden e Hannah (1998), a maioria dos estudos mais antigos utilizou o inglês sinalizado ao invés da American Sign Language - ASL para a adaptação de testes. Posteriormente, esse procedimento foi considerado inadequado, pois a estrutura das LS difere das línguas orais (Braden, 1992; Braden \& Hannah, 1998). No caso do Brasil, significaria traduzir as instruções e itens diretamente para Libras sem as adaptações necessárias, não respeitando a estrutura da língua. Apenas a tradução dos itens para Libras não garante uma adequação satisfatória dos instrumentos, uma vez que podem ser identificados itens carregados de fatores culturais. Por exemplo, questionar a semelhança entre "telefone e rádio", é inadequado, considerando que são aparelhos não utilizados pelos surdos.

Tradicionalmente, a etapa de tradução do instrumento consiste em verter os itens da língua original para a língua nacional (no caso do Brasil, por exemplo, do inglês para o português). No presente estudo, as instruções dos subtestes e os itens verbais foram traduzidos para Libras, pelo pesquisador principal que tem domínio dessa forma de comunicação.

Para tradução, foram consultadas as seguintes fontes: Capovilla e Raphael (2006), Lira e Souza (2008), Capovilla, Raphael e Mauricio (2012). Os itens dos subtestes verbais selecionados para a forma reduzida foram analisados quanto ao seu conteúdo (uso da palavra original e/ou adequabilidade do sinal na comunidade surda) e quanto à possibilidade de tradução para Libras. Foram realizadas exclusões de itens inadequados ou sem sinal correspondente, inclusão de itens novos e, ainda, modificações quando necessárias. Posteriormente, foram feitas adaptações também no Livro de Estímulos, de acordo com as alterações dos subtestes. No processo de adaptação dos itens, foram inseridos itens de exemplo extras nos subtestes Semelhanças e Vocabulário, com base em outros estudos (Braden \& Hannah, 1998; Braden, 2004; Hill-Briggs, Dial, Morere, \& Joyce, 2007; Nunes et al., 2011) que identificaram essa necessidade a fim de assegurar que a criança surda compreenda com maior clareza a tarefa solicitada. Finalizando essa etapa, foi gravado um vídeo do pesquisador sinalizando as consignas e os itens dos subtestes em Libras.

\section{Etapa de Retrotradução e Análise de Juízes}

Maller (1997) assegura que a precisão de uma versão em LS é demonstrada por intermédio de uma tradução reversa. Dessa forma, o vídeo produzido na etapa anterior foi apresentado, individualmente, a sete peritos para que transcrevessem em um protocolo a tradução dos itens para o português. Essa amostra de juízes foi composta por sete especialistas, com idades entre $21 \mathrm{e}$ 44 anos. A maioria era intérprete/tradutor de Libras que atuavam em escolas e universidades. Participou também como juiz, um professor surdo que ministra disciplina de Libras na Universidade. O tempo de experiência e o contato com a comunidade surda variou entre três e 27 anos.

\section{Etapa de Administração da Versão Inicial em Representantes da População-Alvo}

Com o objetivo de testar se a versão inicial do teste está adequada para a população, a literatura aponta a necessidade de uma avaliação qualitativa dos itens e do instrumento como um todo (Jorge, 1998; Pasquali et al., 2010; Borsa, Damásio, \& Bandeira, 2012; Goresnstein, Wang, \& Hungerbühler, 2016). Para tal, a versão inicial do WISC-IV foi aplicada em oito alunos, indicados pelas professoras da única escola especial para surdos da cidade. Concomitantemente, foi aplicado o teste Matrizes Progressivas de Raven - Escala Geral, com o intuito apenas de caracterizar a habilidade cognitiva do grupo.

A versão inicial da Forma Reduzida do WISC-IV foi aplicada de maneira dialogada com objetivo de avaliar o entendimento dos examinandos das instruções e dos itens verbais do teste, assim como os aspectos de operacionalização/logística do estudo. Nessa etapa, a aplicação dos subtestes verbais foi diferente do método usual, não sendo seguidas as orientações referentes ao critério de início e de suspensão, aplicando-se todos os itens de cada subteste. Essa atitude foi tomada uma vez que o nível crescente de dificuldade dos itens, padronizado para ouvintes, provavelmente não seria o mesmo para os surdos.

Considerando-se que a Libras é apreendida pelo canal visual, é importante que o receptor (aplicador) fique atento a todos os sinais manuais concomitantes com as expressões faciais realizadas pelo examinando. Essa 
atenção dificulta o processo simultâneo da percepção e do registro das respostas emitidas. Por essa razão, a aplicação dos subtestes verbais foi filmada (com auxílio de uma câmera digital e um tripé) para posterior transcrição para língua portuguesa, correção e pontuação das respostas.

Após a aplicação, foi realizada uma análise de frequência de acertos por item nos subtestes verbais para avaliar o entendimento dos surdos em relação ao conteúdo dos itens. Também foram identificados os escores mínimos/máximos, média e desvio padrão relativos aos acertos dos participantes em cada subteste.

\section{Resultados e Discussão}

\section{Etapa de Seleção dos Subtestes para Compor a Forma Reduzida}

A FR proposta neste estudo para avaliar surdos com o WISC-IV (FR WISC-IV ${ }^{\mathrm{LB}}$ ) ficou assim constituída: Vocabulário e Semelhanças (Compreensão Verbal), Cubos e Raciocínio Matricial (Organização Perceptual), Código e Procurar Símbolos (Velocidade de Processamento) e Sequência de Números e Letras e Dígitos (Memória Operacional).

Cubos, juntamente com o Vocabulário, foram selecionadas por serem uma díade tradicionalmente utilizada pelos pesquisadores estrangeiros para representar as áreas verbal e não verbal das Escalas Wechsler, por serem as provas que melhor se relacionam com o Fator $\mathrm{g}$ da inteligência (Donders, 1997; Sattler, citado por Donders, 1997; Simões, 2002; Wagner \& cols. 2010). No subteste Vocabulário, assim como no Semelhanças, o tipo de estímulo utilizado é breve por conter questões simples e repetitivas, o que favorece a tradução para Língua de Sinais (Uriel \& Cayssials, 2002). Quanto ao subteste Cubos, as instruções são facilmente traduzidas em LS. Já o Raciocínio Matricial foi escolhido pela facilidade de aplicação e porque "o subteste é relativamente isento de interferências culturais e de domínio do idioma" (Wechsler, 2004, p. 31). Dígitos e Sequência de Números e Letras (IMO) e Código e Procurar Símbolos (IVP) foram selecionados por serem os subtestes principais dos respectivos Índices e por serem facilmente administrados em Libras.

\section{Etapa de Tradução e Adaptação para Libras}

Tentou-se preservar ao máximo as características do teste original, embora várias modificações tenham sido necessárias quanto à exclusão e inclusão de itens, com base nas orientações de Braden (2004). Segundo o autor, as mudanças no processo de avaliação devem atender às deficiências dos indivíduos, desde que não altere o construto medido pelo teste. Tais mudanças são aceitáveis como perspectivas legais e conceituais. Para avaliação de surdos, o autor recomenda ainda a necessidade de modificações relacionadas à inserção de itens de exemplo e treino e de materiais complementares, a aceitação de diferentes formatos de respostas (gestos e escrita), a possibilidade de tempo extra e a não utilização de subtestes saturados de linguagem. A seguir, descrevem-se as alterações realizadas nos subtestes componentes da Forma Reduzida para surdos.

\section{Vocabulário}

$\mathrm{Na}$ versão padronizada para ouvintes, o subteste consiste em 36 itens, apresentados no Livro de Estímulos. Os quatro primeiros compõem figuras a serem nomeadas (Itens Figurativos), os quais foram incluídos para permitir a aplicação na ordem inversa, no caso de erro nos itens iniciais. Tais itens foram incluídos na quarta edição para ampliar o efeito de solo. Os demais itens são lidos e apresentados na forma escrita (duas ou três palavras por página) a fim de que sejam dadas suas definições (Itens Verbais). O subteste não inclui item de exemplo.

$\mathrm{Na}$ forma adaptada para surdos, o subteste Vocabulário ficou constituído por dois itens de exemplo e 29 itens para avaliação, sendo 10 figurativos e 19 verbais. Nessa versão adaptada, foram inseridos dois itens de exemplo: o primeiro - "faca" - foi retirado da primeira versão da Escala para crianças (WISC, 1949). O segundo - "cama" -, retirado do mesmo subteste do WAISIII (Wechsler, 2004). Esses itens foram selecionados por possuírem sinal próprio e por terem abrangência regional (em vários Estados se utiliza o mesmo sinal para essas palavras). Foram acrescentadas figuras a seis itens desse subteste, considerando que o vocabulário se propõe a avaliar as habilidades de nomeação e conceitualização e atendendo às recomendações de Ray (1982), assim como Braden e Hannah (1998), que sugeriram a utilização de outras estratégias visuais para auxiliar na compreensão da tarefa pelos examinandos surdos. Também, o dicionário Novo Deit Libras (Capovilla, Raphael, \& Mauricio, 2009) apresenta de uma a cinco ilustrações (figuras) representativas do sinal em Libras e o vocábulo associado. Segundo os autores, as diversas ilustrações possibilitam que o surdo apreenda diretamente o sentido do sinal. Atendendo a tais considerações, aumentou-se o número de itens que utilizam figuras a serem nomeadas.

Por representarem objetos concretos, foram associadas figuras nos itens 05 (relógio), 06 (chapéu), 07 (guarda-chuva), 08 (vaca), 09 (bicicleta) e 13 (ladrão). Nestes, a sinalização em Libras é a própria resposta e, por isso, foram ampliados os itens que avaliam a capacidade de nomeação e, consequentemente, diminuídos os que mensuram a habilidade de conceitualização. Os demais itens foram exibidos apenas na forma escrita.

Os itens excluídos do subteste Vocabulário foram: 16 (praga); 17 (contra-senso); 23 (exato); 28 (previsão); 29 (unânime); 34 (aberração); 35 (prolixo) e 36 (dilatório). Os itens 16, 17, 28, 34 e 35 não possuem sinal próprio ou um sinal que se aproxime do significado da palavra original. Já o item 23, o sinal de exato abrange todos os 
sinônimos aceitáveis como resposta, sendo utilizado para certo, correto e perfeito. Manter o termo dificultaria as opções para pontuar as respostas. As palavras "unânime" (item 29) e "dilatório" (item 36) possuem sinais correspondentes, recentemente inseridos no Novo Deit Libras. Entretanto, os sinais são próprios da região do Rio de Janeiro, como explicitado no dicionário, sendo pouco conhecidos e utilizados na comunidade surda em geral. No processo de adaptação, quando o vocábulo original não foi localizado, buscou-se outro termo similar com o mesmo significado, encontrado em alguma das referências pesquisadas.

Algumas das palavras excluídas já mencionadas foram consideradas inadequadas no trabalho de Maller (1997) com o WISC-III para surdos. A autora identificou diversos problemas no subteste Vocabulário: vinte itens não tinham sinal correspondente e necessitaram ser digitalizados (soletrados); cinco itens (corajoso, fábula, exato, unânime e compelir) demonstraram-se inadequados para avaliação de surdos; dois itens (aberração e dilatório) nenhum sujeito surdo pontuou e seis itens (ladrão, mímica, árduo, isolar, rivalidade e aflição) apresentaram um Funcionamento Diferencial do Item (DIF) contra os sujeitos surdos, indicando que estes não avaliam, com a mesma precisão, surdos e ouvintes.

\section{Semelhanças}

$\mathrm{Na}$ versão para ouvintes, esse subteste é constituído por um item de exemplo e 23 itens apresentados apenas oralmente pelo aplicador. Os itens consistem em díades de palavras que representam objetos ou conceitos comuns para que o examinando indique suas semelhanças. $\mathrm{Na}$ forma adaptada, esse subteste ficou composto por três itens de exemplo e 18 itens para avaliação, sendo 13 itens figurativos e cinco itens verbais.

Em Semelhanças, foram feitas modificações substanciais. Foram acrescentados dois itens de exemplo: "bola" e "roda" e "vela" e "lâmpada", ambos retirados da versão precedente do instrumento, o WISC-III (Figueiredo, 2001), os quais foram excluídos da quarta edição. As palavras utilizadas nos três itens de exemplo possuem sinal próprio em todas as fontes consultadas.

Para todas as díades de palavras que se referiam a objetos concretos foram incluídas, no Livro de Estímulos, figuras com as respectivas palavras escritas abaixo delas (Itens Figurativos). Os demais itens foram apresentados apenas sob a forma escrita, também impressos no Livro de Estímulos (itens verbais). Essas alterações estão embasadas em Ray (1982) e Braden e Hannah (1998) que sugeriram o máximo de utilização de recursos visuais para auxiliar os surdos na compreensão da tarefa. Tal possibilidade também foi considerada por Wechsler (1991) quando se referiu à utilização do teste com surdos. A ordem dos itens foi alterada, iniciando-se pelos figurativos (objetos concretos).

Foram excluídos cinco itens: 13 (poeta e pintor); 20 (vingança e perdão); 21 (permissão e restrição); 22 (realidade e sonho) e 23 (espaço e tempo), ou por não terem sinais em Libras, ou pelo fato de que os sinais correspondentes alteram a proposta do item. O sinal para "poeta" é inexistente nas fontes consultadas. Já a palavra "pintor" possui um sinal correspondente na versão antiga do Dicionário Enciclopédico Trilíngue da Língua Brasileira de Sinais. Entretanto, na versão mais atual (Novo Deit Libras), este vocábulo foi excluído, demonstrando a ausência da sua utilização. A díade "vingança" e "perdão" não têm sinais correspondentes. Existem sinais para "vingar/vingar-se" e "perdoar", entretanto, também não são comumente utilizados na comunidade surda. O sinal utilizado para "permissão" é o mesmo de "livre" e a palavra "restrição" não possui sinal correspondente. O sinal para "realidade" foi recentemente inserido no Novo Deit Libras, porém é representado com o mesmo sinal utilizado para designar "verdade". "Sonho" também carece de um sinal próprio, sendo apresentado nas fontes consultadas somente "sonhar", o que iria alterar a ideia principal do item. A palavra "espaço", com o significado exigido no teste original, não possui sinal próprio; em Libras, o termo utilizado refere-se a espaço físico. Já o termo "tempo" possui sinal próprio, mas com sentido de "período/horas/época”.

Em função das adaptações feitas nos subtestes Vocabulário e Semelhanças, foram necessárias modificações no Livro de Estímulos. Foram impressas as novas figuras dos subtestes verbais e separados os itens-palavra do vocabulário (um por página, ao invés de agrupamentos de duas e três palavras).

\section{Dígitos e Sequência de Número e Letras}

Os itens foram apenas traduzidos para Libras, utilizando-se a digitalização manual para aplicação. É importante ressaltar que, em ambas as provas, o construto originalmente envolvido (memória auditiva) é substituído, considerando que os examinandos visualizam as sinalizações e, após reproduzem a sequência de sinais, acionando a função de memória visual (Wechsler, 1991; Braden, 2004).

\section{Cubos, Raciocínio Matricial, Código e Procurar Símbolos}

Não foram feitas modificações na estrutura dos itens que formam os Fatores Organização Perceptual e Velocidade de Processamento. Apenas as consignas foram traduzidas e adaptadas para Libras.

\section{Etapa de Retrotradução e Análise de Juízes}

No subteste Vocabulário, os dois itens de exemplo e 16 itens foram aceitos por unanimidade pelos juízes. Quanto aos demais itens, a maioria das sugestões dadas pelos peritos referiu-se a expressões faciais e configurações de mãos. No subteste Semelhanças, a instrução para ouvinte utiliza a pergunta "Qual a semelhança entre...?". $\mathrm{Na}$ versão adaptada para surdos, os juízes sugeriram que 
inserir a sinalização de "quase igual", seria mais adequado para ser sinalizado em Libras.

A maioria das sugestões dos especialistas referiu-se a melhoramentos dos sinais já utilizados. Todas as sugestões dadas pelos juízes, consideradas pertinentes, foram gravadas em vídeo pelos próprios profissionais-especialistas. Posteriormente, os itens alterados foram regravados pelo pesquisador na versão final do vídeo.

\section{Etapa de Administração da Versão Inicial em Representantes da População-Alvo}

Oito alunos, com grau de surdez entre moderado e profundo, usuários da Libras responderam os instrumentos, aplicados de forma dialogada. A maioria dos sujeitos era do sexo masculino $(N=5)$ e estavam cursando entre o $4^{\circ}$ e o $8^{\circ}$ ano do ensino fundamental. As idades dos examinandos variaram entre 11 e 16 anos $(M=13,6$; $D P=1,69)$ e tinham em torno de dois anos de atraso entre idade/escolaridade. Quanto ao resultado no teste Matrizes Progressivas de Raven, administrado junto com a versão inicial FR WISC-IV ${ }^{\mathrm{LB}}$, a maioria dos participantes $(N=5)$ obteve percentil abaixo da média. Tais resultados podem estar associados ao fato de a escola ser pública e atender famílias mais carentes, com pouca abertura para aprendizagem da Libras e dificuldades de acesso à recursos para estimulação precoce dos filhos surdos.

A Tabela 1 apresenta os escores brutos obtidos pelo grupo de alunos surdos, em cada subteste da forma reduzida. No caso dos subtestes Vocabulário e Semelhanças, observa-se o número de itens administrados na forma adaptada e os escores máximos recalculados, considerando-se a modificação na quantidade dos itens. No caso do primeiro, a versão original é formada por 36 itens, com pontuação máxima de 68 (itens 1 a $4=1$ ponto; itens $5 \mathrm{a}$ $36=2$ pontos). No caso do subteste Semelhanças, a versão original constitui-se de 23 itens, com escore máximo de 44 pontos (itens 1 e $2=1$ ponto; itens 3 a $23=2$ pontos). Na versão adaptada, a pontuação diferenciada nos itens iniciais foi mantida; entretanto, o número de itens foi diminuído e, consequentemente, seu escore máximo.

Tabela 1

Escores Brutos Observados na Amostra

\begin{tabular}{|c|c|c|c|c|c|}
\hline Subteste & $\begin{array}{l}\text { Número de } \\
\text { Itens }\end{array}$ & $\begin{array}{l}\text { Escore } \\
\text { Máximo }\end{array}$ & $\begin{array}{c}\text { Mínimo da } \\
\text { Amostra }\end{array}$ & $\begin{array}{c}\text { Máximo } \\
\text { da Amostra }\end{array}$ & Média (DP) \\
\hline Cubos & 14 & 68 & 13 & 39 & $26,25(8,92)$ \\
\hline Vocabulário & $29^{*}$ & 54 & 20 & 33 & $26,50(5,04)$ \\
\hline Código (Forma B) & 119 & 119 & 30 & 45 & $39,38(4,75)$ \\
\hline Semelhanças & $18^{*}$ & 34 & 01 & 16 & $10,38(5,04)$ \\
\hline Rac. Matricial & 35 & 35 & 14 & 25 & $19,75(3,88)$ \\
\hline Seq. de Números e Letras & 10 & 30 & 0 & 16 & $9,38(6,35)$ \\
\hline Proc. Símbolos (Forma B) & 60 & 60 & 11 & 25 & $18,00(5,32)$ \\
\hline Dígitos & 16 & 32 & 07 & 15 & $11,88(2,70)$ \\
\hline
\end{tabular}

Nota. $\left({ }^{*}\right)$ Versão adaptada

Apesar de o subteste Vocabulário ter necessitado maiores adaptações, como já foi observado em trabalhos anteriores com o WISC-III com surdos (Maller, 1997), em relação ao percentual de acertos (dois ou um ponto), 26 itens foram respondidos corretamente por $50 \%$ da amostra. Já no subteste Semelhanças, somente nove itens atingiram esse percentual. Os dados sugerem que o subteste Vocabulário teve mais itens de dificuldade mediana do que o Semelhanças.

A maior dificuldade em responder os itens do subteste Semelhanças, necessariamente não indica que a tradução dos itens foi menos satisfatória. Deve-se levar em conta que, segundo Conceição e Figueiredo (2012), esse subteste é o de maior dificuldade para crianças com déficit cognitivo - característica da amostra estudada, segundo o teste Matrizes Progressivas de Raven - por terem raciocínio mais concreto.

Pode-se concluir que a tradução dos itens dos subtestes verbais foi satisfatória, uma vez que todos os itens foram respondidos corretamente por pelo menos um aluno, demonstrando compreensão. Nos demais subtestes não houve dificuldades para entender as instruções e as tarefas. A proposta da versão experimental da forma reduzida em Libras parece adequada para avaliar a capacidade intelectual de surdos. Novos estudos devem dar continuidade ao processo de adaptação em busca de evidências psicométricas e normas.

\section{Considerações Finais}

O presente estudo propôs a apresentação de uma Forma Reduzida do teste WISC-IV para surdos (FR WISC-IV ${ }^{\mathrm{LB}}$ ), buscando a utilização de um teste padrão-ouro para avaliar a inteligência na sua versão mais recente, permitindo uma aplicação rápida e útil para rastreio além de possibilitar a avaliação cognitiva de um grupo especial. Esse artigo descreve, com detalhes, a etapa dos procedimentos teóricos do processo de adaptação transcultural, 
no caso, adaptação de um teste direcionado a ouvintes para escolares surdos. Na seleção dos subtestes para compor a forma reduzida - Vocabulário, Semelhanças, Cubos, Raciocínio Matricial, Código, Procurar Símbolos, Sequencia de Números e Letras e Dígitos - teve-se a preocupação em manter o modelo quadrifatorial do teste, além de oferecer aos psicólogos a possibilidade de avaliar a habilidade verbal dos surdos, enquanto que na literatura, a maior parte dos trabalhos encontrados, priorizam a estimativa da habilidade não verbal.

As várias modificações nos subtestes Vocabulário e Semelhanças da forma adaptada para surdos foram necessárias, principalmente, pelas seguintes razões: (a) não foram encontrados sinais próprios para diversas palavras utilizadas nos itens dos subtestes verbais da versão original para ouvintes e (b) as línguas de sinais empregam representações para traduzir ideias e, assim, muitos termos não têm equivalência com a palavra expressa ou escrita pelo ouvinte. Por esta razão, diversos itens foram excluídos dos subtestes verbais, uma vez que, segundo Maller (1997), os sujeitos surdos são pertencentes a uma minoria linguística e podem estar menos familiarizados com os conteúdos dos itens do teste projetado para ouvintes. Entretanto, deve-se considerar que a Libras é recente e está em pleno desenvolvimento. Por exemplo, para a palavra "balde" (item
04 do Vocabulário) não existia um sinal específico nos dicionários. Entretanto, no Novo Deit Libras (Capovilla et al., 2012), foi inserido um sinal correspondente, demonstrando a constante atualização da língua.

Dentre as limitações deste estudo, pode-se apontar que um maior número de juízes, incluindo mais surdos e, também, surdos nativos (filhos de pais também surdos), de regiões diferentes, poderia ampliar o repertório de opções para a tradução dos itens para Libras. Em relação à aplicação dialogada nos representantes da população-alvo, os participantes constituíram apenas o extrato mais alto da população quanto à série escolar; e, ao mesmo tempo, constituiu-se do mais baixo quanto à capacidade cognitiva, fator que pode ter limitado a contribuição deles para a etapa da análise teórica dos itens.

O objetivo do presente estudo foi propor uma forma reduzida do WISC-IV adaptada para surdos. Desenvolveram-se todas as etapas relacionadas a analise teórica dos itens, propondo-se uma versão experimental, que deverá ser ainda avaliada em uma amostra representativa. Entretanto, é importante ressaltar que, no caso de utilização do teste com surdos de outros Estados, deve haver um novo estudo de readequação de alguns sinais representantes dos termos, considerando as especificidades regionais e culturais.

\section{Referências}

Anastasi, A., \& Urbina, S. (2000). Testes para Populações Especiais. Em A. Anastasi e S. Urbina (Ed.), Testagem Psicológica (202-229) Porto Alegre: Artes Médicas Sul.

Borsa, J. C., Damásio, B. F., \& Bandeira, D. R. (2012). Adaptação e validação de instrumentos psicológicos entre culturas: Algumas considerações. Paidéia, 22(53).

Braden, J. P. (1992). Intellectual assessment of deaf and hard-of-hearing people: A quantitative and qualitative research synthesis. School Psychology Review, 21(1), 82-94.

Braden, J. P. (2004). Hard-of-hearing and deaf clients: using the wisc-iv with clients who are hard-of-hearing or deaf. Em A. Prifitera (Ed.), WISC-IV Clinical Use and Interpretation: Scientist-Practitioner Perspectives (351-380). San Diego: Academic Press.

Braden, J. P., \& Hannah, J. M. (1998). Assessment of hearing-impaired and deaf children with the WISC-III. Em A. Prifitera e D. H. Saklofske (Ed.) WISC-III Clinical Use and Interpretation (175-201). San Diego: Academic Press.

Capovilla, F. C., \& Raphael, W. D. (2006). Dicionário enciclopédico ilustrado trilíngue da Língua de Sinais Brasileira. Volumes 1 e 2. (2a . Ed.) São Paulo: EDUSP.

Capovilla, F. C., Raphael, W. D., \& Mauricio, A. C. L. (2012). Novo Deit-Libras: Dicionário Enciclopédico Ilustrado Trilíngue da Língua de Sinais Brasileira (Libras) baseado em Linguística e Neurociências Cognitivas. Volumes 1 e 2. São Paulo: EDUSP.

Conceição, M. B. O., \& Figueiredo, V. L. M. (2012). Habilidades cognitivas de crianças com deficiência intelectual. Em V. L. M., Figueiredo, J. M. G. Araújo \& F. A. Vidal (Eds.). Avaliando com o WISC-III: Prática e Pesquisa (pp. 163-176). São Paulo: Casa do Psicólogo.

Coutinho, A. C. A. M., \& Nascimento, E. (2010). Formas Abreviadas do WAIS-III para Avaliação da Inteligência. Avaliação Psicológica, 9(1), 25-33.

Crawford, J. R., Anderson, V, Rankin, P. M., \& MacDonald, J. (2010). An index-based short-form of the WISC-IV with accompanying analysis of the reliability and abnormality of differences. British Journal of Clinical Psychology, 49, 235-258.

Donders, J. (1997). A short form of the WISC-III for clinical use. Psychological Assessment, vol. 9(1), 15-20.

Dumont, R., \& Faro C. A. (1993). WISC-III short form for learning-disabled students. Psychology in the Schools, 30, $212-219$.

Figueiredo, V. L. M. (2001). Uma adaptação brasileira do teste de inteligência WISC-III. (Tese de doutorado não publicada). Curso de PósGraduação em Psicologia, Universidade de Brasília, Brasília, DF.

Goldfeld, M. (1997). A criança surda: Linguagem e cognição numa perspectiva sócio-interacionista. São Paulo: Plexus.

Gorenstein, C., Wang, Y-P., Hungerbühler, I. (2016). Instrumentos de avaliação em saúde mental. Porto Alegre: Artmed.

Hill-Briggs, F., Dial, J. G., Morere, \& D. A., Joyce, A. (2007) Neuropsychological assessment of persons with physical disability, visual impairment or blindness, and hearing impairment or deafness. Archives of Clinical Neuropsychology, 22, 389-404.

Jorge, M. R. (1998). Adaptação transcultural de instrumentos de pesquisa em saúde mental. Rev. Psiquiatr. Clín. (São Paulo), 25(5), 233-9. 
Krouse, H. E. (2008) The reliability and validity of the WISC-IV with deaf and hard-of-hearing children (Dissertação de mestrado). Graduate Faculty of North Carolina State University. Raleigh, North Carolina.

Lira, G. A. \& Souza, T. A. F. (2008) LIBRAS - Dicionário da Língua Brasileira de Sinais - Versão 2.1. Recuperado de http://www.acessobrasil. org.br/libras/

Lubbadeh, J. (2006). Línguas Silenciosas. Revista Mente e cérebro, Editora Duetto, Ano XIV, nº165, 79-83.

Maller, S. J. (1994). Validity and item bias of the WISC-III with deaf children (Tese de doutorado). Faculdade de Psicologia da Educação. The University of Arizona.

Maller, S. J. (1997) Deafness and WISC-III item difficulty: Invariance and Fit. Journal of School Psychology, 35(3), $299-314$.

Marchesi, A. (2004) Desenvolvimento e educação das crianças surdas. Em C. Coll, A. Marchesi \& J. Palacios (Ed.), Desenvolvimento Psicológico e Educação. 2a . ed., vol. 3, (171-192) Porto Alegre: Artmed.

Nunes, T., Evans, D., Barros, R., \& Burman, D. (2011). Promovendo o sucesso das crianças surdas em matemática: Uma intervenção precoce. XIII Conferencia Interamericana de Educación Matemática. Comité Interamericano de Educación Matemática - CIAEM. Recife, Brasil. Recuperado de http://www.cimm.ucr.ac.cr/ocs/index.php/xiii_ciaem/xiii_ciaem/paper/viewFile/2840/1155

Pasquali, L. et al. (2010). Instrumentação psicológica: fundamentos e práticas. Porto Ālegre: Artmed.

Quadros, R. M. (1997). Educação de surdos: A aquisição da linguagem. Porto Alegre: Artes Médicas.

Ray, S. (1982). Adapting the WISC-R for deaf children. Assessment for Effective Intervention, 7(3), 147-157. Recuperado de http://aei.sagepub. com/content $/ 7 / 3 / 147$.short

Ryan, J. J., Glass, L. A., \& Brown, C. N. (2007). Administration time estimates for Wechsler Intelligence Scale for Children-IV subtests, composites, and short forms. Journal of Clinical Psychology, 63(4), 309-318.

Simões, M. R. (2002). Utilizações da WISC-III na avaliação neuropsicológica de crianças e adolescentes. Paidéia, 12(23), 11-23.

Uriel, F., \& Cayssials, A. (2002). Disfunciones sensoriales: Sordera. Em A. Cayssials (Ed.), WISC-III Nuevas Investigaciones. Normas da la Ciudad de Buenos Aires y el conurbano bonaerense. Cuadernos de evaluación psicológica (77-95). Buenos Aires: Paidós.

Vechia, S. G. D. (2011). Formas Reduzidas do WISC-III: características psicométricas de quatro modelos (Dissertação de mestrado). Universidade Católica de Pelotas, Pelotas. Recuperado de http:/www.ucpel.tche.br/ppgsaude/dissertacoes/Mestrado/2011/FORMAS\%20 REDUZIDAS\%20DO\%20WISC-III-\%20CARACTER\%CDSTICAS\%20-\%20Shana\%20Gularte\%20Della\%20Vechia.pdf

Vidal, F. A. S., Figueiredo, V. L. M., \& Nascimento, E. (2011). A Quarta Edição do WISC Americano. Avaliação Psicológica, 10(2), $205-207$.

Wagner, F., Pawlowski, J., Yates, D. B., Camey, S. A., \& Trentini, C. M. (2010). Viabilidade da estimativa de QI a partir dos subtestes Vocabulário e Cubos da WAIS-III. Psico-USF, 15(2), 215-224.

Wechsler, D. (1991). Wechsler intelligence scale for children - Third Edition (WISC-III): Manual. San Antonio: Psychological Corporation.

Wechsler, D. (2004) Escala de Inteligência Wechsler para Adultos (WAIS-III): Manual. Adaptação e padronização de uma amostra brasileira. São Paulo: Casa do Psicólogo.

Wechsler, D. (2013). Escala Wechsler de Inteligência para Crianças (WISC-IV). $4^{\mathrm{a}}$ ed. Manual técnico. Tradução do manual original. São Paulo: Casa do Psicólogo.

\section{Sobre os autores}

Tharso de Souza Meyer é Psicólogo (UCPel), Especialista em Saúde Mental e Atenção Psicossocial (ESP/SC) e em Terapia CognitivoComportamental (Unyleya), Mestre e Doutorando no Programa de Pós-Graduação em Saúde e Comportamento (UCPel). Bolsista da Coordenação de Aperfeiçoamento de Pessoal de Nível Superior (CAPES).

Vera Lúcia Marques de Figueiredo é Psicóloga, graduada pela Universidade Católica de Pelotas (UCPel), pós-graduada no mestrado em Psicologia da Pontifícia Universidade Católica do Rio Grande do Sul (PUC-RS) e no doutorado em Psicologia da Universidade de Brasília. Atua como docente de graduação e de pós-graduação na UCPel desde 1980. 